ALEA, Lat. Am. J. Probab. Math. Stat. 15, 49-66 (2018)

DOI: 10.30757/ALEA.v15-03

\title{
On some integral estimates for solutions of stochastic equations driven by symmetric stable processes
}

\author{
Vladimir P. Kurenok \\ Department of Electrical and Systems Engineering, \\ Washington University in St. Louis, \\ One Brookings Drive, St. Louis, MO 63130-4899, USA \\ E-mail address: kurenokv@ese.wustl.edu
}

\begin{abstract}
Let $X$ be a solution of stochastic differential equation $d X_{t}=b\left(X_{t-}\right) d Z_{t}+$ $\gamma|b|^{\alpha}\left(X_{t}\right) d t, t \geq 0$ where $Z$ is a one-dimensional symmetric stable process of index $0<\alpha \leq 2$ and let $\tau_{m}(X)=\inf \left\{t \geq 0:\left|X_{t}\right| \geq m\right\}, m \in \mathbb{Z}_{+}$. We prove various $L_{p}$-estimates for processes $X$ for $p=1,2$. In particular, it is shown that, if $\gamma \neq 0$ and $0<\alpha \leq 2$, then for all $t>0$ and a measurable function $f: \mathbb{R} \rightarrow[0, \infty]$, it holds $\mathbf{E} \int_{0}^{t \wedge \tau_{m}(X)}|b|^{\alpha}\left(X_{s}\right) f\left(X_{s}\right) d s \leq N\|f\|_{2, m}$ where $\|f\|_{2, m}$ is the $L_{2}$-norm of the function $f$ on the interval $[-m, m]$ and the constant $N$ depends on $\alpha, m, \gamma$, and $t$ only. For $\gamma=0$ and $1 / 2<\alpha \leq 2$, similar $L_{p}$-estimates with $p=1,2$ are proven. As an application, we use obtained estimates to prove the existence of (weak) solutions for corresponding stochastic differential equations with $\gamma \neq 0$ and $\gamma=0$.
\end{abstract}

\section{Introduction}

It is known that the integral estimates of Krylov type play an important role in the theory of stochastic processes and its applications. In particular, they provide a powerful method when establishing the existence of (weak) solutions for corresponding stochastic differential equations and are one of the main tools by proving the existence of optimal strategies in control problems associated with the SDEs. To be more precise, let $f: \mathbb{R} \rightarrow[0, \infty]$ be a measurable function and define

$$
\|f\|_{p, m}=\left(\int_{-m}^{m}|f|^{p}(y) d y\right)^{1 / p}
$$

Received by the editors August 9th, 2016; accepted November 25th, 2017.

2010 Mathematics Subject Classification. 60H10, 60J60, 60J65, 60G44.

Key words and phrases. Krylov's estimates, symmetric stable processes, one-dimensional stochastic differential equations, weak convergence. 
to be its $L_{p}$-norm on the interval $[-m, m], m \in \mathbb{Z}_{+}, p \geq 1$. For a stochastic process $\left(X_{t}\right), t \geq 0$ defined on a given probability space $(\Omega, F, P)$, the estimates of the form

$$
\mathbf{E} \int_{0}^{t \wedge \tau_{m}(X)} e^{-\phi_{s}} \Phi_{s} f\left(X_{s}\right) d s \leq N\|f\|_{p, m}
$$

where $\left(\phi_{t}\right)$ and $\left(\Phi_{t}\right)$ are nonnegative processes to be specified and

$$
\tau_{m}(X)=\inf \left\{t \geq 0:\left|X_{t}\right| \geq m\right\}
$$

are called the Krylov's estimates. The inequalities (1.1) were first proved by Krylov (1980) (see chapter 2) in the case when $X$ is a diffusion process, in other words when $X$ satisfies the equation

$$
d X_{t}=b\left(t, X_{t}\right) d W_{t}+a\left(t, X_{t}\right) d t
$$

where $W$ is a Brownian motion.

Many authors have tried to generalize the estimates to other classes of processes $X$ different from the diffusion ones. For example, in Mel'nikov (1983) one derived the estimates of the form (1.1) for some classes of continuous semimartingales $X$. Some generalizations of Krylov's estimates for diffusion processes with jumps were obtained by Anulova and Pragarauskas (1977) and Lepeltier and Marchal (1976). We refer also to Situ (2005), chapter 4 for another formulation of Krylov's estimates for a class of processes with jumps. However, it should be noticed that all mentioned generalizations of Krylov's estimates for processes with jumps have one property in common: they are derived for processes $X$ being not purely discontinuous, that is when $X$ is of the form

$$
X_{t}=X_{0}+\int_{0}^{t} b\left(s, X_{s}\right) d W_{s}+\int_{0}^{t} a\left(s, X_{s}\right) d s+\int_{0}^{t} \int c\left(s, X_{s-}, y\right) \tilde{N}(d s, d y), t \geq 0
$$

where $\tilde{N}(d t, d y)$ is a martingale measure generated by a driven process and $b \neq 0$. As the result, the tools utilized to prove the estimates for corresponding processes were similar to those used by Krylov and the presence of the term with the diffusion coefficient $b(t, x) \neq 0$ was crutial.

There is less known about Krylov's estimates for purely discontinuous processes $X$. Pragarauskas and Zanzotto (2000) proved a variant of Krylov's estimates for the case when $X$ is a solution of the stochastic differential equation

$$
d X_{t}=b\left(t, X_{t-}\right) d Z_{t}
$$

where $Z$ is a symmetric stable process of index $1<\alpha<2$. More precisely, they proved that, for any $m \in \mathbb{Z}_{+}$and $t \geq 0$, it holds

$$
\mathbf{E} \int_{0}^{t \wedge \tau_{m}(X)}\left|b\left(s, X_{s}\right)\right|^{\alpha / 2} f\left(s, X_{s}\right) d s \leq N\|f\|_{2, m, t}=: N\left(\int_{0}^{t} \int_{-m}^{m} f^{2}(s, y) d s d y\right)^{1 / 2} .
$$

In the case of $Z$ being a symmetric stable process with $1<\alpha<2$ and $X$ satisfying the equation

$$
d X_{t}=b\left(X_{t-}\right) d Z_{t}+a\left(X_{t}\right) d t
$$

the corresponding Krylov's estimates of the form

$$
\mathbf{E} \int_{0}^{t \wedge \tau_{m}(X)}\left|b\left(X_{s}\right)\right|^{\alpha} f\left(X_{s}\right) d s \leq N\|f\|_{2, m}
$$


were proven in Kurenok (2008a) assuming $|a(x)| \leq K|b(x)|^{\alpha}$ for all $x \in \mathbb{R}$ and a constant $K>0$. We also mention Kurenok (2008b) where the results from Kurenok (2008a) were extended to the the SDE (1.3) with $Z$ being a Cauchy process, that is a symmetric stable process with $\alpha=1$.

It is worth to notice that all mentioned results do not include estimates for the case when $Z$ is a symmetric stable process of index $\alpha<1$ and one of our main goals here is the proof of some variants of Krylov's estimates covering the case with $\alpha<1$. In other words, we shall establish here several integral estimates of the form (1.4) when $X$ satisfies the equation

$$
d X_{t}=b\left(X_{t-}\right) d Z_{t}+\gamma|b|^{\alpha}\left(X_{t}\right) d t, \quad t \geq 0, \gamma \in \mathbb{R}, \quad X_{0}=x_{0} \in \mathbb{R}
$$

where $Z$ ia a symmetric stable process. For $\gamma=0$, the estimates will hold for $1 / 2<\alpha \leq 2$ while for $\gamma \neq 0$ they will be true for all $0<\alpha \leq 2$.

As an application of the obtained integral estimates, we will prove the existence of weak solutions for the equation (1.5) with $\gamma \neq 0$ and $\gamma=0$.

We briefly recall some known existence results for the equation (1.5).

If $\alpha=2$, the process $Z$ is a Brownian motion, the only one process in the class of symmetric stable processes with continuous trajectories and finite variance. The equation (1.5) in this case was studied in details by Engelbert and Schmidt (1991) who, in particular, were able to find nessecary and sufficient conditions for the existence of solutions when $\gamma=0$. In other words, they had shown that the equation (1.5) with $\gamma=0$ has a non-trivial solution for any initial value $x_{0} \in \mathbb{R}$ if and only if $b^{-2} \in L^{l o c}(\mathbb{R})$, that is the function $b^{-2}$ is locally integrable on $\mathbb{R}$.

For $\alpha \in(0,2)$, the equation (1.5) with $\gamma=0$ was studied by Zanzotto (1997, 2002). For the values of $1<\alpha<2$, he was able to generalize the existence result of Engelbert and Schmidt replacing the condition $b^{-2} \in L^{l o c}(\mathbb{R})$ by the condition $|b|^{-\alpha} \in L^{l o c}(\mathbb{R})$. For the values of $0<\alpha \leq 1$, he proved the existence of solutions under the following sufficient conditions:

1) There exists a number $\delta>1$ such that $|b|^{-\delta} \in L^{l o c}(\mathbb{R})$;

2) There exists a number $U>0$ such that $l\left(B_{U}\right)<\infty$, where

$$
B_{U}=\{y \in \mathbb{R}:|b(y)|>U\}
$$

and $l$ is the Lebesgue measure on $\mathbb{R}$ (cf. Theorem 3.3 in Zanzotto, 1997).

In this note, in particular, sufficient conditions for the existence of solutions of the equation (1.5) without drift are given which are different from conditions 1) and 2) found by Zanzotto. Moreover, for the values of $0<\alpha<1 / 2$, our integrability condition $|b|^{-2 \alpha} \in L^{l o c}$ is weaker than the integrability condition in 1$)$.

\section{Preliminary facts}

By $\mathbf{D}_{[0, \infty)}(\mathbb{R})$ we denote, as usual, the Skorokhod space, i.e. the set of all realvalued functions $z:[0, \infty) \rightarrow \mathbb{R}$ with right-continuous trajectories and with finite left limits (also called cádlág functions). For simplicity, we shall write D instead of $\mathbf{D}_{[0, \infty)}(\mathbb{R})$. We will equip $\mathbf{D}$ with the $\sigma$-algebra $\mathcal{D}$ generated by the Skorokhod topology. Under $\mathbf{D}^{n}$ we will understand the $n$-dimensional Skorokhod space defined as $\mathbf{D}^{n}=\mathbf{D} \times \cdots \times \mathbf{D}$ with the corresponding $\sigma$-algebra $\mathcal{D}^{n}$ being the direct product of $n$ one-dimensional $\sigma$-algebras $\mathcal{D}$.

Let $(\Omega, \mathcal{F}, P)$ be a complete probability space carrying a process $Z$ with $Z_{0}=0$ and let $\mathbf{F}=\left(\mathcal{F}_{t}\right)$ be a filtration on $(\Omega, \mathcal{F}, P)$. The notation $(Z, \mathbf{F})$ means that $Z$ 
is adapted to $\mathbf{F}$. We call $(Z, \mathbf{F})$ a symmetric stable process of index $\alpha \in(0,2]$ if trajectories of $Z$ belong to $\mathbf{D}$ and

$$
\mathbf{E}\left(\exp \left(i \xi\left(Z_{t}-Z_{s}\right)\right) \mid \mathcal{F}_{s}\right)=\exp \left(-(t-s)|\xi|^{\alpha}\right)
$$

for all $t>s \geq 0$ and $\xi \in \mathbb{R}$.

For all $0<\alpha \leq 2, Z$ is a Markov process and can be described in terms of characteristics of Markov processes. Thus, for any function $f \in L^{\infty}(\mathbb{R})$ and $t \geq 0$, define the operator

$$
\left(P_{t} f\right)(x):=\int_{\Omega} f\left(x+Z_{t}\right) d P(\omega)
$$

where $L^{\infty}(\mathbb{R})$ is the Banach space of functions $f: \mathbb{R} \rightarrow \mathbb{R}$ with the norm $\|f\|_{\infty}=$ ess $\sup |f(x)|$. The sequence $\left(P_{t}\right)_{t \geq 0}$ is called the family of convolution operators associated with $Z$. For a suitable class of functions $g(x)$, we can define so-called infinitesimal generator $\mathcal{L}$ of the process $Z$ as

$$
(\mathcal{L} g)(x)=\lim _{t \downarrow 0} \frac{\left(P_{t} g\right)(x)-g(x)}{t} .
$$

On the other hand, in the case of $\alpha \in(0,2), Z$ is a purely discontinuous Markov process that can be described by its Poisson jump measure (jump measure of $Z$ on interval $[0, t])$ defined as

$$
N(U \times[0, t])=\sum_{s \leq t} 1_{U}\left(Z_{s}-Z_{s-}\right),
$$

the number of jumps of $Z$ on the interval $[0, t]$ whose size lies in the set $U$. The compensating measure of $N$, say $\hat{N}$, is given (see, for example, Appelbaum, 2009, p. 32) by

$$
\hat{N}(U)=\mathbf{E} N(U \times[0,1])=\int_{U} \frac{c_{\alpha}}{|x|^{1+\alpha}} d x,
$$

where $c_{\alpha}$ is a suitable constant. One defines then $\tilde{N}(d s, d y):=N(d s, d y)-\hat{N}(d s, d y)$ called the martingale measure associated with the process $Z$.

It is known that the operator $\mathcal{L}$ can be written as

$$
(\mathcal{L} g)(x)=\int_{\mathbb{R} \backslash\{0\}}\left[g(x+z)-g(x)-\mathbf{1}_{\{|z|<1\}} g^{\prime}(x) z\right] \frac{c_{\alpha}}{|z|^{1+\alpha}} d z
$$

defined on $g \in C^{2}(\mathbb{R})$, where $C^{2}(\mathbb{R})$ is the set of all bounded and twice continuously differentiable functions $g: \mathbb{R} \rightarrow \mathbb{R}$. In the case of $\alpha=2$, the infinitesimal generator of a Brownian motion process is the second derivative operator.

For $1<\alpha<2$, the operator in (2.2) has the form

$$
(\mathcal{L} g)(x)=\int_{\mathbb{R} \backslash\{0\}}\left[g(x+z)-g(x)-g^{\prime}(x) z\right] \frac{c_{\alpha}}{|z|^{1+\alpha}} d z
$$

since in this case

$$
\int_{|z| \geq 1} z \frac{c_{\alpha}}{|z|^{1+\alpha}} d z=0
$$


Using the introduced measures, the equation (1.3) is then understood as

$X_{t}=x_{0}+\int_{0}^{t} a\left(X_{s}\right) d s+\int_{0}^{t} \int_{|y| \leq 1} b\left(X_{s-}\right) y \tilde{N}(d s, d y)+\int_{0}^{t} \int_{|y|>1} b\left(X_{s-}\right) y N(d s, d y)$.

For $b=1$ and $a=0$, the relation (2.3) represents the famous Lévy-Itô decomposition of a symmetric stable process as a particular case of a Lévy process.

Since for all $0<\alpha<2$, the process $X$ of the form (2.3) is a semimartingale, the Itô formula applies so that for any $t \geq 0$ and any function $g \in C^{2}(\mathbb{R})$ such that $g$ and $g^{\prime}$ are bounded, it follows that (see Ikeda and Watanabe, 1981, chapter 2)

$$
\begin{aligned}
g\left(X_{t}\right)-g\left(X_{0}\right)= & \int_{0}^{t} g^{\prime}\left(X_{s}\right) a\left(X_{s}\right) d s+\int_{0}^{t} \int_{|y| \geq 1}\left[g\left(X_{s-}+y b\left(X_{s-}\right)\right)-g\left(X_{s-}\right)\right] N(d s, d y) \\
& +\int_{0}^{t} \int_{|y|<1}\left[g\left(X_{s-}+y b\left(X_{s-}\right)\right)-g\left(X_{s-}\right)\right] \tilde{N}(d s, d y)+ \\
& \int_{0}^{t} \int_{|y|<1}\left[g\left(X_{s-}+y b\left(X_{s-}\right)\right)-g\left(X_{s-}\right)-y b\left(X_{s-}\right) g^{\prime}\left(X_{s-}\right)\right] \frac{c_{\alpha} d s d y}{|y|^{1+\alpha}} .
\end{aligned}
$$

In the case of $1<\alpha<2$, the above formula becomes

$$
\begin{gathered}
g\left(X_{t}\right)-g\left(X_{0}\right)=\int_{0}^{t} g^{\prime}\left(X_{s}\right) a\left(X_{s}\right) d s+\int_{0}^{t} \int\left[g\left(X_{s-}+y b\left(X_{s-}\right)\right)-g\left(X_{s-}\right)\right] \tilde{N}(d s, d y)+ \\
\int_{0}^{t} \int\left[g\left(X_{s-}+y b\left(X_{s-}\right)\right)-g\left(X_{s-}\right)-y g^{\prime}\left(X_{s-}\right)\right] \frac{c_{\alpha} d s d y}{|y|^{1+\alpha}}
\end{gathered}
$$

and making the change of variables $z=y b\left(X_{s-}\right)$ in the last integral will simplify it further to the form

$$
\begin{gathered}
g\left(X_{t}\right)-g\left(X_{0}\right)=\int_{0}^{t} g^{\prime}\left(X_{s}\right) a\left(X_{s}\right) d s+\int_{0}^{t} \int\left[g\left(X_{s-}+y b\left(X_{s-}\right)\right)-g\left(X_{s-}\right)\right] \tilde{N}(d s, d y)+ \\
\int_{0}^{t}|b|^{\alpha}\left(X_{s}\right) \mathcal{L} g\left(X_{s}\right) d s
\end{gathered}
$$

where the term

$$
\int_{0}^{t} \int\left[g\left(X_{s-}+y b\left(X_{s-}\right)\right)-g\left(X_{s-}\right)\right] \tilde{N}(d s, d y)
$$

is a martingale.

In a similar way, in the case of $0<\alpha<1$, the Itô's formula is written as $g\left(X_{t}\right)-g\left(X_{0}\right)=\int_{0}^{t} g^{\prime}\left(X_{s}\right) a\left(X_{s}\right) d s+\int_{0}^{t} \int\left[g\left(X_{s-}+y b\left(X_{s-}\right)\right)-g\left(X_{s-}\right)\right] N(d s, d y)$ so that taking the expectation from both sides of the last relation we obtain

$$
\begin{gathered}
\mathbf{E}\left[g\left(X_{t}\right)-g\left(X_{0}\right)\right]= \\
\mathbf{E} \int_{0}^{t} g^{\prime}\left(X_{s}\right) a\left(X_{s}\right) d s+\mathbf{E} \int_{0}^{t} \int\left[g\left(X_{s-}+y b\left(X_{s-}\right)\right)-g\left(X_{s-}\right)\right] \frac{c_{\alpha} d s d y}{|y|^{1+\alpha}} .
\end{gathered}
$$

Again, by making change of variables $z=y b\left(X_{s-}\right)$ in the last integral, it is not hard to see that it holds

$$
\mathbf{E}\left[g\left(X_{t}\right)-g\left(X_{0}\right)\right]=\mathbf{E} \int_{0}^{t} g^{\prime}\left(X_{s}\right) a\left(X_{s}\right) d s+\mathbf{E} \int_{0}^{t}|b|^{\alpha}\left(X_{s}\right) \mathcal{L} g\left(X_{s}\right) d s .
$$


The case of $\alpha=1$ can be treated the same way as it is a mixture between cases $0<\alpha<1$ and $1<\alpha<2$. We summarize the obtained results in

Proposition 2.1. Assume that $X$ is of the form (1.3) and $g \in C^{2}(\mathbb{R})$ such that $g$ and $g^{\prime}$ are bounded. Then, for any $0<\alpha \leq 2$, it holds

$$
\mathbf{E}\left[g\left(X_{t}\right)-g\left(X_{0}\right)\right]=\mathbf{E} \int_{0}^{t} g^{\prime}\left(X_{s}\right) a\left(X_{s}\right) d s+\mathbf{E} \int_{0}^{t}|b|^{\alpha}\left(X_{s}\right) \mathcal{L} g\left(X_{s}\right) d s .
$$

The following result about the convergence of stochastic integrals with respect to symmetric stable processes is proven in Pragarauskas and Zanzotto (2000), Lemma 2.3. For the sack of completeness of our exposition, we formulate it here. The result is originally due to A. V. Skorokhod who proved it for the case of Brownian motion and it is often called the Skorokhod's lemma about the convergence of stochastic integrals (cf. Krylov, 1980, Lemma 2, p. 87).

Proposition 2.2. Let $Z^{n}, n=0,1,2, \ldots$ be a sequence of symmetric stable processes of the same index $0<\alpha \leq 2$ defined on a complete probability space $(\Omega, \mathcal{F}, P)$ with a filtration $\mathbf{F}$. Assume that $g^{n}, n=0,1,2, \ldots$ is a sequence of predictable processes defined on the same probability space so that the stochastic integrals $\int_{0}^{t} g_{s}^{n} d Z_{s}^{n}$ are well defined for all $n=0,1,2, \ldots$ and $t \geq 0$. Moreover, let the following conditions be satisfied:

1) for all $t \geq 0$,

$$
Z_{t}^{n} \rightarrow Z_{t}^{0}, g_{t}^{n} \rightarrow g_{t}^{0}
$$

in probability as $n \rightarrow \infty$;

2) for all $t \geq 0$,

$$
\lim _{N \rightarrow \infty} \sup _{n} P\left(\sup _{0 \leq s \leq t}\left|g_{s}^{n}\right|>N\right)=0
$$

3) for all $t \geq 0$ and $\epsilon>0$,

$$
\lim _{r \downarrow 0} \varlimsup_{n \rightarrow \infty} \sup _{0 \leq t_{1}<t_{2} \leq t, t_{2}-t_{1} \leq r} P\left(\left|g_{t_{2}}^{n}-g_{t_{1}}^{n}\right|>\epsilon\right)=0 .
$$

Then, for all $t \geq 0$, it holds

$$
\int_{0}^{t} g_{s}^{n} d Z_{s}^{n} \rightarrow \int_{0}^{t} g_{s}^{0} d Z_{s}
$$

in probability as $n \rightarrow \infty$.

We notice finally that the use of Fourier transforms can simplify calculations when working with the operator $\mathcal{L}$. Let $g \in L_{1}(\mathbb{R})$ and

$$
\hat{g}(\xi):=\int_{\mathbb{R}} e^{i z \xi} g(z) d z
$$

be the Fourier transform of $g$. The following fact will be used later (cf. Proposition 2.1 in Kurenok, 2008a).

Proposition 2.3. Let $\mathcal{L}$ be the infinitesimal generator of a symmetric stable process $Z$ with $0<\alpha \leq 2$ and assume that $g \in C^{2}(\mathbb{R}), \mathcal{L} g \in L_{1}(\mathbb{R})$. Then

$$
\widehat{(\mathcal{L} g})(\xi)=-|\xi|^{\alpha} \hat{g}(\xi) \text { and } \widehat{g^{\prime}}(\xi)=i \xi \hat{g}(\xi) \text {. }
$$




\section{Some integral estimates}

In this section we assume that $Z$ is a symmetric stable process of index $0<$ $\alpha \leq 2$ defined on a probability space $(\Omega, \mathcal{F}, P)$ with a filtration $\mathbf{F}$ and $\gamma \in \mathbb{R}$ is a fixed constant.

For all $x \in \mathbb{R}$ and a nonnegative, measurable function $f \in C_{0}^{\infty}(\mathbb{R})^{1}$, let

$$
v(x):=\mathbf{E} \int_{0}^{\infty} e^{-s} f\left(x+Z_{s}+\gamma s\right) d s .
$$

Since $\left(Z_{t}+\gamma t\right), t \geq 0$ is a Feller process, the formula of Dynkin (cf. Kallenberg, 1997, Lemma 17.21) will imply that, for all $t \geq 0$, it holds

$$
v(x)=\mathbf{E}\left\{\int_{0}^{t} e^{-s} f\left(x+Z_{s}+\gamma s\right) d s+e^{-t} v\left(x+Z_{t}+\gamma t\right)\right\} .
$$

Applying Itô's formula to the expression $e^{-t} v\left(x+Z_{t}+\gamma t\right)$, we obtain

$$
\mathbf{E} e^{-t} v\left(x+Z_{t}+\gamma t\right)=v(x)+\mathbf{E} \int_{0}^{t} e^{-s}\left[\mathcal{L} v-v+\gamma v_{x}\right]\left(x+Z_{s}+\gamma s\right) d s
$$

where $v_{x}$ denotes the derivative of $v$. Substituting the formula (3.1) into the last relation yields

$$
\mathbf{E} \int_{0}^{t} e^{-s}\left[\mathcal{L} v-v+\gamma v_{x}+f\right]\left(x+Z_{s}+\gamma s\right) d s .
$$

Dividing this equation by $t$ and letting $t$ tend to zero, we obtain (see also Krylov, 1980, ch. 1, pp. 2-4)

$$
\mathcal{L} v(x)-v(x)+\gamma v_{x}(x)+f(x)=0
$$

where the obtained equation holds a.e. in $\mathbb{R}$.

Equation (3.2) is also known in the control theory as the Bellman equation.

Lemma 3.1. a) Let $\gamma \neq 0$ and $0<\alpha \leq 2$. Then, for all $x \in \mathbb{R}$, it holds

$$
v(x) \leq M\|f\|_{2}:=M\left(\int_{\mathbb{R}} f^{2}(y) d y\right)^{1 / 2},
$$

where the constant $M$ depends on $\alpha$ and $\gamma$ only.

b) Let $\gamma=0$ and $1 / 2<\alpha \leq 2$. Then, for all $x \in \mathbb{R}$, it holds

$$
v(x) \leq M\|f\|_{2},
$$

where the constant $M$ depends on $\alpha$ only.

c) Let $1<\alpha<2$ and $\gamma \neq 0$. Then, for all $x \in \mathbb{R}$, it holds

$$
v(x) \leq M\|f\|_{1}:=M\left(\int_{\mathbb{R}}|f|(y) d y\right),
$$

where the constant $M$ depends on $\alpha$ and $\gamma$ only.

\footnotetext{
${ }^{1} C_{0}^{\infty}(\mathbb{R})$ denotes the class of all infinitely differentiable real-valued functions with compact support defined on $\mathbb{R}$
} 
Proof: For any function $h: \mathbb{R} \rightarrow \mathbb{R}$ such that $h \in L_{1}(\mathbb{R})$ and any $\varepsilon>0$ we define

$$
h^{(\varepsilon)}(x)=\frac{1}{\varepsilon} \int_{\mathbb{R}} h(y) q\left(\frac{x-y}{\varepsilon}\right) d y
$$

to be the $\varepsilon$-convolution of $h$ with a smooth function $q$ such that $q \in C_{0}^{\infty}(\mathbb{R})$ and $\int_{\mathbb{R}} q(x) d x=1$.

After taking the $\varepsilon$-convolution on both sides of equation (3.2), we obtain

$$
\mathcal{L} v^{(\varepsilon)}(x)-v^{(\varepsilon)}(x)+\gamma v_{x}^{(\varepsilon)}(x)+f^{(\varepsilon)}(x)=0 .
$$

Applying Proposition 2.3 and the Parseval identity to the relation

$$
\left(\mathcal{L} v^{(\varepsilon)}(x)-v^{(\varepsilon)}(x)+\gamma v_{x}^{(\varepsilon)}(x)\right)^{2}=\left(f^{(\varepsilon)}(x)\right)^{2},
$$

yields

$$
\int_{\mathbb{R}}\left|\hat{v}^{(\varepsilon)}(\xi)\right|^{2}\left[\left(|\xi|^{\alpha}+1\right)^{2}+\gamma^{2} \xi^{2}\right] d \xi=\int_{\mathbb{R}}\left|\hat{f}^{(\varepsilon)}(\xi)\right|^{2} d \xi .
$$

Assume that

$$
M_{1}:=\int_{\mathbb{R}} \frac{d \xi}{\left(|\xi|^{\alpha}+1\right)^{2}+\gamma^{2} \xi^{2}} .
$$

Clearly, for $\gamma=0$ and $1 / 2<\alpha \leq 2$, the constant $M_{1}$ is finite and depends on $\alpha$ only. Similarly, if $\gamma \neq 0$ and $0<\alpha \leq 2$, it follows that $M_{1}<\infty$ and $M_{1}$ depends on $\alpha$ and $\gamma$ only.

Using the identity (3.7) and the inverse Fourier transform we obtain for all $x \in \mathbb{R}$

$$
\begin{gathered}
\left(v^{(\varepsilon)}(x)\right)^{2} \leq \\
\frac{M_{1}}{4 \pi^{2}} \int_{\mathbb{R}}\left|\hat{v}^{(\varepsilon)}(\xi)\right|^{2}\left[\left(|\xi|^{\alpha}+1\right)^{2}+\gamma^{2} \xi^{2}\right] d \xi \leq \\
\frac{M_{1}}{4 \pi^{2}} \int_{\mathbb{R}}\left(f^{(\varepsilon)}(z)\right)^{2} d z .
\end{gathered}
$$

The claims a) and b) follow then by taking the limit $\varepsilon \rightarrow 0$ in the above inequality and using the Lebesgue dominated convergence theorem.

To prove c), we notice that for any function $f$ such that $\hat{f}$ exists it holds for all $\xi \in \mathbb{R}$

$$
|\hat{f}(\xi)| \leq \int_{-\infty}^{\infty}|f|(x) d x
$$

so that

$$
\sup _{\xi \in \mathbb{R}}|\hat{f}(\xi)| \leq\|f\|_{1}
$$

Again, the application of the inverse Fourier transform yields for all $x \in \mathbb{R}$ and $1<\alpha<2$

$$
v(x) \leq \frac{1}{2 \pi} M_{1}\|f\|_{1}
$$

where

$$
M_{1}=\int_{-\infty}^{\infty} \frac{d \xi}{\sqrt{\left.|\xi|^{(\alpha}+1\right)^{2}+\gamma^{2} \xi^{2}}}<\infty
$$

The argument with the Lebesgue dominated convergence theorem finishes then the proof. 
Remark 3.2. The addition of the term $\gamma t(\gamma \neq 0)$ to the process $Z$ allows to extend the analytical estimate of the sup-norm of the function $v(x)$ through the $L_{2}$-norm of the function $f(x)$ from the range $1 / 2<\alpha \leq 2$ to the entire range $0<\alpha \leq 2$ of the stability index. In this sense the addition of drift plays a "regularizing effect" on the estimates.

The analytical estimates established can be used to derive corresponding integral estimates for solutions of stochastic differential equations driven by symmetric stable processes.

Theorem 3.3. a) Assume $0<\alpha \leq 2$ and $X$ is a solution of equation (1.5) with $\gamma \neq 0$. Then, for any $t>0, m \in \mathbb{Z}_{+}, x_{0} \in[-m, m]$, and any measurable function $f: \mathbb{R} \rightarrow[0, \infty]$, one has

$$
\mathbf{E} \int_{0}^{t \wedge \tau_{m}(X)} e^{-\phi_{s}}|b|^{\alpha}\left(X_{s}\right) f\left(x_{0}+X_{s}\right) d s \leq M\|f\|_{2, m},
$$

where $\phi_{t}=\int_{0}^{t}|b|^{\alpha}\left(X_{s}\right) d s$ and $M$ is a constant depending on $\alpha, \gamma, m$, and $t$ only.

b) Assume that $1 / 2<\alpha \leq 2$ and $X$ is a solution of equation (1.5) with $\gamma=0$. Then, for any $t>0, m \in \mathbb{Z}_{+}, x_{0} \in[-m, m]$, and any measurable function $f: \mathbb{R} \rightarrow$ $[0, \infty]$, one has

$$
\mathbf{E} \int_{0}^{t \wedge \tau_{m}(X)} e^{-\phi_{s}}|b|^{\alpha}\left(X_{s}\right) f\left(x_{0}+X_{s}\right) d s \leq M\|f\|_{2, m},
$$

where $\phi_{t}$ is defined in a) and $M$ is a constant depending on $\alpha, m$, and $t$ only.

c) Assume that $1<\alpha \leq 2$ and $X$ is a solution of equation (1.5) with $\gamma \in \mathbb{R}$. Then, for any $t>0, m \in \mathbb{Z}_{+}, x_{0} \in[-m, m]$, and any measurable function $f: \mathbb{R} \rightarrow$ $[0, \infty]$, one has

$$
\mathbf{E} \int_{0}^{t \wedge \tau_{m}(X)} e^{-\phi_{s}}|b|^{\alpha}\left(X_{s}\right) f\left(x_{0}+X_{s}\right) d s \leq M\|f\|_{1, m},
$$

where $\phi_{t}$ is defined as above, $M$ is a constant depending on $\alpha, m$, and $t$ only, and $\|f\|_{1, m}$ defines the $L_{1}$-norm of $f$ on the interval $[-m, m]$.

Proof: The proofs of all three statements a), b), and c) are similar and they use the corresponding estimates a), b), and c) from Lemma 3.1, respectively. We only prove part a).

Let $f \in C_{0}^{\infty}(\mathbb{R})$ and $v$ be a solution of equation (3.2) satisfying the inequality (3.3). As before, we apply the convolution to equation (3.2) to obtain the relation $(3.6)$.

Then, for all $s \in\left[0, t \wedge \tau_{m}(X)\right)$, applying the Itô's formula to the expression

$$
v^{(\varepsilon)}\left(x_{0}+X_{s}\right) e^{-\phi_{s}}
$$

and using Proposition 2.1, yields

$$
\begin{gathered}
\mathbf{E} v^{(\varepsilon)}\left(x_{0}+X_{s}\right) e^{-\phi_{s}}-v^{(\varepsilon)}\left(x_{0}\right)= \\
\mathbf{E} \int_{0}^{s} e^{-\phi_{u}}|b|^{\alpha}\left(X_{u}\right)\left(\mathcal{L} v^{(\varepsilon)}-v^{(\varepsilon)}+\gamma v_{x}^{(\varepsilon)}\right)\left(x_{0}+X_{u}\right) d u= \\
-\mathbf{E} \int_{0}^{s} e^{-\phi_{u}}|b|^{\alpha}\left(X_{u}\right) f^{(\varepsilon)}\left(x_{0}+X_{u}\right) d u .
\end{gathered}
$$


By Lemma 3.1, part a) one has that

$$
\mathbf{E} \int_{0}^{s \wedge \tau_{m}(X)} e^{-\phi_{u}}|b|^{\alpha}\left(X_{u}\right) f^{(\varepsilon)}\left(x_{0}+Z_{u}\right) d u \leq \sup _{x_{0}} v^{(\varepsilon)}\left(x_{0}\right) \leq M\left\|f^{(\varepsilon)}\right\|_{2, m} .
$$

It remains to pass to the limit in the above inequality letting $\varepsilon \rightarrow 0, s \rightarrow t$, and using the Fatou's lemma.

The inequality (3.8) can be extended in a standard way first to any function $f \in L_{2}(\mathbb{R})$ and then to any non-negative, measurable function using the monotone class theorem arguments (see, for example, Dellacherie and Meyer, 1980, Theorem $20)$.

Corollary 3.4. a) Let $0<\alpha \leq 2$ and $X$ be a solution of the equation (1.5) with $\gamma \neq 0$. Assume that there exists a constant $K>0$ such that $\frac{1}{K}<|b(x)|<K$ for all $x \in \mathbb{R}$. Then, for all $t>0, m \in \mathbb{Z}_{+}$, and any measurable function $f: \mathbb{R} \rightarrow[0, \infty]$, it holds

$$
\mathbf{E} \int_{0}^{t \wedge \tau_{m}(X)} f\left(X_{s}\right) d s \leq M\|f\|_{2, m}
$$

where the constant $M$ depends on $K, \alpha, \gamma, m$, and $t$ only.

b) Let $1 / 2<\alpha \leq 2$ and $X$ be a solution of the equation (1.5) with $\gamma=0$. Assume that there exists a constant $K>0$ such that $\frac{1}{K}<|b(x)|<K$ for all $x \in \mathbb{R}$. Then, for all $t>0, m \in \mathbb{Z}_{+}$, and any measurable function $f: \mathbb{R} \rightarrow[0, \infty]$, it holds

$$
\mathbf{E} \int_{0}^{t \wedge \tau_{m}(X)} f\left(X_{s}\right) d s \leq M\|f\|_{2, m}
$$

where the constant $M$ depends on $K, \alpha, m$, and $t$ only.

c) Let $1<\alpha \leq 2$ and $X$ be a solution of the equation (1.5) with $\gamma=0$. Assume that there exists a constant $K>0$ such that $\frac{1}{K}<|b(x)|<K$ for all $x \in \mathbb{R}$. Then, for all $t>0, m \in \mathbb{Z}_{+}$, and any measurable function $f: \mathbb{R} \rightarrow[0, \infty]$, it holds

$$
\mathbf{E} \int_{0}^{t \wedge \tau_{m}(X)} f\left(X_{s}\right) d s \leq M\|f\|_{1, m}
$$

where the constant $M$ depends on $K, \alpha, m$, and $t$ only.

Proof: The estimates a), b), and c) of Corollary 3.4 follow directly from the corresponding estimates of Theorem 3.3 by noticing that

$$
\begin{gathered}
\int_{0}^{t \wedge \tau_{m}(X)} f\left(X_{s}\right) d s=\int_{0}^{t \wedge \tau_{m}(X)} e^{\phi_{s}}|b|^{-\alpha}\left(X_{s}\right) e^{-\phi_{s}}|b|^{\alpha}\left(X_{s}\right) f\left(X_{s}\right) d s \leq \\
M_{3} \int_{0}^{t \wedge \tau_{m}(X)} e^{-\phi_{s}}|b|^{\alpha}\left(X_{s}\right) f\left(X_{s}\right) d s
\end{gathered}
$$

where $e^{\phi_{s}}|b|^{-\alpha}\left(X_{s}\right) \leq M_{3}$ for all $s \in[0, t]$ and the constant $M_{3}$ depends on $K, \alpha, m$, and $t$ only.

As a particular case of the estimates in Corollary 3.4, with $b=1$, we obtain the following integral estimates for symmetric stable processes with drift.

Corollary 3.5. a) Let $Z$ be a symmetric stable process of index $0<\alpha \leq 2$ and $\gamma \neq 0$. Then, for all $t>0, m \in \mathbb{Z}_{+}$, and any measurable function $f: \mathbb{R} \rightarrow[0, \infty]$, it holds

$$
\mathbf{E} \int_{0}^{t \wedge \tau_{m}(Z)} f\left(Z_{s}+\gamma s\right) d s \leq M\|f\|_{2, m}
$$


where the constant $M$ depends on $\alpha, m, \gamma$, and $t$ only.

b) Let $Z$ be a symmetric stable process of index $1 / 2<\alpha \leq 2$. Then, for all $t>0, m \in \mathbb{Z}_{+}$, and any measurable function $f: \mathbb{R} \rightarrow[0, \infty]$, it holds

$$
\mathbf{E} \int_{0}^{t \wedge \tau_{m}(Z)} f\left(Z_{s}\right) d s \leq M\|f\|_{2, m}
$$

where the constant $M$ depends on $\alpha, m$, and $t$ only.

c) Let $Z$ be a symmetric stable process of index $1<\alpha \leq 2$. Then, for all $t>0, m \in \mathbb{Z}_{+}$, and any measurable function $f: \mathbb{R} \rightarrow[0, \infty]$, it holds

$$
\mathbf{E} \int_{0}^{t \wedge \tau_{m}(Z)} f\left(Z_{s}\right) d s \leq M\|f\|_{1, m}
$$

where the constant $M$ depends on $\alpha, m$, and $t$ only.

\section{Existence of weak solutions}

Here we are going to apply the obtained integral estimates to prove the existence of (weak) solutions for corresponding stochastic differential equations.

We recall that the equation (1.5) is said to have a (weak) solution with initial value $x_{0} \in \mathbb{R}$ if there exist a probability space $(\Omega, \mathcal{F}, P)$ with a filtration $\mathbf{F}$ on it and cádlág stochastic processes $(X, \mathbf{F})$ and $(Z, \mathbf{F})$ such that $Z$ is a symmetric stable process of the given index $\alpha$ and it holds

$$
X_{t}=x_{0}+\int_{0}^{t} b\left(X_{s-}\right) d Z_{s}+\gamma \int_{0}^{t}|b|^{\alpha}\left(X_{s}\right) d s, \quad t \geq 0 \quad P \text {-a.s. }
$$

As mentioned before, the stochastic integral in (4.1) can be understood by means of semimartingale integration theory. On the other hand, as it was shown in Rosiński and Woyczyński (1986), the stochastic integral in (4.1) can be defined similarly as for the Brownian motion case $(\alpha=2)$ following some isometry relations between corresponding $L_{\alpha}$ spaces. One of the consequences of such definition is the following property of a stable integral valid for all $\alpha \in(0,2]$ : there exist constants $C_{1}$ and $C_{2}$ depending on $\alpha$ only such that for all $t>0$

$$
C_{1} \mathbf{E} \int_{0}^{t}\left|\sigma_{s}\right|^{\alpha} d s \leq \sup _{\lambda>0} \lambda^{\alpha} P\left(\sup _{s \leq t}\left|\int_{0}^{s} \sigma_{u} d Z_{u}\right|>\lambda\right) \leq C_{2} \mathbf{E} \int_{0}^{t}\left|\sigma_{s}\right|^{\alpha} d s
$$

where $\left(\sigma_{t}\right)$ is a suitable integrand such that the stochastic integral exists.

Theorem 4.1. Let $0<\alpha<2$ and $\gamma \neq 0$. Assume that there exists a constant $K>0$ such that $|b(x)| \leq K$ for all $x \in \mathbb{R}$ and $|b|^{-2 \alpha} \in L^{l o c}(\mathbb{R})$. Then, for any $x_{0} \in \mathbb{R}$, there exists a solution of the equation (1.5).

Proof: Step 1. First, we assume additionally that the function $b(x)$ is also bounded away from zero. Without loss of generality, we can assume that $\frac{1}{K} \leq|b| \leq K$.

Then, since $b$ is bounded and so $|b|^{\alpha}$, we can find, again without loss of generality, a sequence of functions $b_{n}, n \geq 1$, such that all $b_{n}$ and $\left|b_{n}\right|^{\alpha}$ are globally Lipshitz continuous and uniformly bounded by the constant $K$. Moreover, $b_{n} \rightarrow b$ and $\left|b_{n}\right|^{\alpha} \rightarrow|b|^{\alpha}$ as $n \rightarrow \infty$ pointwise and in $\|\cdot\|_{2, m}$-norm for all $t>0, m \in \mathbb{Z}_{+}$. It is then well-known that, for any $n=1,2, \ldots$, there exists a unique pathwise solution for the equation (1.5). That is, for any fixed symmetric stable process $Z$ 
defined on a probability space $(\Omega, \mathcal{F}, P)$ with a filtration $\mathbf{F}$, there exists a sequence of processes $X^{n}$ such that (cf. Theorem 6.2.3 in Appelbaum, 2009)

$$
X_{t}^{n}=x_{0}+\int_{0}^{t} b_{n}\left(X_{s-}^{n}\right) d Z_{s}+\gamma \int_{0}^{t}\left|b_{n}\right|^{\alpha}\left(X_{s}^{n}\right) d s \quad t \geq 0 \quad P \text {-a.s. }
$$

Let $Y_{t}^{n}:=\int_{0}^{t} b_{n}\left(X_{s-}^{n}\right) d Z_{s}+\gamma \int_{0}^{t}\left|b_{n}\right|^{\alpha}\left(X_{s}^{n}\right) d s$. Next, we are going to verify that the sequence of processes $\left(X^{n}, Z\right), n \geq 1$ is tight in the sense of weak convergence in $\left(\mathbf{D}^{2}, \mathcal{D}^{2}\right)$. Clearly, it suffices to verify that the sequence $Y^{n}$ is tight. Due to the Aldous' criterion (Aldous, 1978), we have only to show that

$$
\lim _{N \rightarrow \infty} \varlimsup_{n \rightarrow \infty} P\left(\sup _{0 \leq s \leq t}\left|Y_{s}^{n}\right|>N\right)=0
$$

for all $t \geq 0$ and

$$
\varlimsup_{n \rightarrow \infty} P\left(\left|Y_{t \wedge\left(\tau^{n}+r_{n}\right)}^{n}-Y_{t \wedge \tau^{n}}^{n}\right|>\varepsilon\right)=0
$$

for all $t \geq 0, \varepsilon>0$, every sequence of $\mathbf{F}$-stopping times $\tau^{n}$, and every sequence of real numbers $r_{n}$ such that $r_{n} \downarrow 0$.

From the property (4.2) and the uniform boundedness of coefficients $b_{n}$ and $\left|b_{n}\right|^{\alpha}$, it follows immediately that both conditions (4.4) and (4.5) are satisfied.

Since the sequence $\left\{X^{n}, Z\right\}$ is tight, there exists a subsequence $\left\{n_{k}\right\}, k=$ $1,2, \ldots$, a probability space $(\bar{\Omega}, \overline{\mathcal{F}}, \bar{P})$ and the process $(\bar{X}, \bar{Z})$ on it with values in $\left(\mathbf{D}^{2}, \mathcal{D}^{2}\right)$ such that $\left(X^{n_{k}}, Z\right)$ converges weakly (in distribution) to the process $(\bar{X}, \bar{Z})$ as $k \rightarrow \infty$. For simplicity, let $\left\{n_{k}\right\}=\{n\}$.

According to the well-known embedding principle of Skorokhod (see, e.g. Theorem 2.7, ch. 1 in Ikeda and Watanabe, 1981), there exists a probability space $(\tilde{\Omega}, \tilde{\mathcal{F}}, \tilde{P})$ and the processes $(\tilde{X}, \tilde{Z}),\left(\tilde{X}^{n}, \tilde{Z}^{n}\right), \quad n=1,2, \ldots$, on it such that

i) $\left(\tilde{X}^{n}, \tilde{Z}^{n}\right) \rightarrow(\tilde{X}, \tilde{Z})$ as $n \rightarrow \infty \tilde{P}$-a.s.

ii) $\left(\tilde{X}^{n}, \tilde{Z}^{n}\right)=\left(X^{n}, Z\right)$ in distribution for all $n=1,2, \ldots$

By using standard measurability arguments (Krylov, 1980, chapter 2, p. 89), the processes $\tilde{Z}^{n}$ and $\tilde{Z}$ will be processes with independent increments with respect to the augmented filtrations $\tilde{\mathbf{F}}^{n}$ and $\tilde{\mathbf{F}}$ generated by processes $\left(\tilde{X}^{n}, \tilde{Z}^{n}\right)$ and $(\tilde{X}, \tilde{Z})$, respectively. Because the finite-dimensional distributions of $\left(\tilde{X}^{n}, \tilde{Z}^{n}\right)$ and $\left(X^{n}, Z\right)$ coincide, it is then easy to see that the property (2.1) is satisfied so that $\tilde{Z}^{n}$ and $\tilde{Z}$ are in fact symmetric stable processes of the same index $\alpha$ as the process $Z$ is.

The properties i), ii) and the equation (4.3) imply that (cf. Krylov, 1980, chapter 2, p. 89 and Pragarauskas and Zanzotto, 2000, Lemma 3.2)

$$
\tilde{X}_{t}^{n}=x_{0}+\int_{0}^{t} b_{n}\left(\tilde{X}_{s}^{n}\right) d \tilde{Z}_{s}^{n}+\gamma \int_{0}^{t}\left|b_{n}\right|^{\alpha}\left(\tilde{X}_{s}^{n}\right) d s, \quad t \geq 0, \quad \tilde{P} \text {-a.s. }
$$

On the other hand, the same properties and the quasi-left continuity of the processes $\tilde{X}^{n}$ yield

$$
\lim _{n \rightarrow \infty} \tilde{X}_{t}^{n}=\tilde{X}_{t} \quad \tilde{P}_{\text {-a.s. }}
$$

Therefore, in order to show that the process $\tilde{X}$ is a solution of the equation (1.5), it suffices to verify that, for all $t \geq 0$,

$$
\lim _{n \rightarrow \infty} \int_{0}^{t} b_{n}\left(\tilde{X}_{s}^{n}\right) d \tilde{Z}_{s}^{n}=\int_{0}^{t} b\left(\tilde{X}_{s}\right) d \tilde{Z}_{s} \quad \tilde{P} \text { - a.s. }
$$


and

$$
\lim _{n \rightarrow \infty} \int_{0}^{t}\left|b_{n}\right|^{\alpha}\left(\tilde{X}_{s}^{n}\right) d s=\int_{0}^{t}|b|^{\alpha}\left(\tilde{X}_{s}\right) d s \quad \tilde{P} \text { - a.s. }
$$

It is clear that the relation (4.8) will follow from the relation (4.7) which remains only one to prove.

The following fact provides the corresponding integral estimate for the limiting process $\tilde{X}$ and can be proven similar as Lemma 4.2 in Kurenok (2008a).

Lemma 4.2. For any Borel measurable function $f: \mathbb{R} \rightarrow[0, \infty)$ and any $t \geq 0$, there exists a sequence $m_{k} \in(0, \infty), k=1,2, \ldots$ such that $m_{k} \uparrow \infty$ as $k \rightarrow \infty$ and it holds

$$
\tilde{\mathbf{E}} \int_{0}^{t \wedge \tau_{m_{k}}(\tilde{X})} f\left(\tilde{X}_{s}\right) d s \leq M\|f\|_{2, m_{k}}
$$

where the constant $M$ depends on $K, \alpha, t$, and $m_{k}$ only.

Without loss of generality, we can assume in the lemma above that $\left\{m_{k}\right\}=$ $\{m\}$. Now, to prove (4.7), it is enough to verify that, for all $t \geq 0$ and $\varepsilon>0$, we have

$$
\lim _{n \rightarrow \infty} \tilde{P}\left(\left|\int_{0}^{t} b_{n}\left(\tilde{X}_{s}^{n}\right) d \tilde{Z}_{s}^{n}-\int_{0}^{t} b\left(\tilde{X}_{s}\right) d \tilde{Z}_{s}\right|>\varepsilon\right)=0 .
$$

In order to prove (4.10) we estimate for a fixed $k \in \mathbb{Z}_{+}$that

$$
\begin{gathered}
\tilde{P}\left(\left|\int_{0}^{t} b_{n}\left(\tilde{X}_{s}^{n}\right) d \tilde{Z}_{s}^{n}-\int_{0}^{t} b\left(\tilde{X}_{s}\right) d \tilde{Z}_{s}\right|>\varepsilon\right) \leq \\
\tilde{P}\left(\left|\int_{0}^{t} b_{k}\left(\tilde{X}_{s}^{n}\right) d \tilde{Z}_{s}^{n}-\int_{0}^{t} b_{k}\left(\tilde{X}_{s}\right) d \tilde{Z}_{s}\right|>\frac{\varepsilon}{3}\right) \\
+\tilde{P}\left(\left|\int_{0}^{t \wedge \tau_{m}\left(\tilde{X}^{n}\right)}\left[b_{k}-b_{n}\right]\left(\tilde{X}_{s}^{n}\right) d \tilde{Z}_{s}^{n}\right|>\frac{\varepsilon}{3}\right) \\
+\tilde{P}\left(\left|\int_{0}^{t \wedge \tau_{m}(\tilde{X})}\left[b_{k}-b\right]\left(\tilde{X}_{s}\right) d \tilde{Z}_{s}\right|>\frac{\varepsilon}{3}\right)+\tilde{P}\left(\tau_{m}\left(\tilde{X}^{n}\right)<t\right)+\tilde{P}\left(\tau_{m}(\tilde{X})<t\right)= \\
I_{n, k}^{1}+I_{n, k, m}^{2}+I_{k, m}^{3}+\tilde{P}\left(\tau_{m}\left(\tilde{X}^{n}\right)<t\right)+\tilde{P}\left(\tau_{m}(\tilde{X})<t\right) .
\end{gathered}
$$

Since, for any fixed $k$, the function $b_{k}$ is bounded and Lipshitz continuous, the processes $g_{t}^{n}=b_{k}\left(\tilde{X}_{t}^{n}\right), n=1,2, \ldots$ and $g_{t}^{0}=b_{k}\left(\tilde{X}_{t}\right)$ satisfy the assumptions of Proposition 2.2 so that $I_{n, k}^{1} \rightarrow 0$ as $n \rightarrow \infty$.

To show that $I_{n, k, m}^{2} \rightarrow 0$ as $n \rightarrow \infty$ and $I_{k, m}^{3} \rightarrow 0$ as $k \rightarrow \infty$, we use first the inequality (4.2) and then the Corollary 3.4 and Lemma 4.2, respectively, to obtain

and

$$
I_{n, k, m}^{2} \leq \varepsilon^{-\alpha} M\left\|b_{k}-b_{n}\right\|_{2, m}
$$

$$
I_{k, m}^{3} \leq \varepsilon^{-\alpha} M\left\|b_{k}-b\right\|_{2, m}
$$

where the constant $M$ depends on $K, \alpha, m$, and $t$ only. Since $\left\|b_{n}-b\right\|_{2, m} \rightarrow 0$ as $n \rightarrow \infty$, we have that the right-hand sides in (4.11) and (4.12) converge to 0 by letting first $n \rightarrow \infty$ and then $k \rightarrow \infty$.

Because of the property $\tau_{m}\left(\tilde{X}^{n}\right) \rightarrow \tau_{m}(\tilde{X})$ as $n \rightarrow \infty \tilde{P}$-a.s.,

$$
\tilde{P}\left(\tau_{m}\left(\tilde{X}^{n}\right)<t\right) \rightarrow \tilde{P}\left(\tau_{m}(\tilde{X})<t\right) \quad \text { as } \quad n \rightarrow \infty
$$


for all $m \in \mathbb{Z}_{+}, t>0$. Therefore, the last two terms can be made arbitrarly small by choosing large enough $m$ for all $n$ due to the fact that the processes $\tilde{X}^{n}$ and $\tilde{X}$ satisfy the property (4.4). This proves (4.10).

Hence we have shown that $\tilde{X}$ is a solution of the equation (1.5) with a symmetric stable process $\tilde{Z}$.

Step 2. Now we drop the additional assumption of $b$ being bounded from below assumed in Step 1.

The main lines of the proof are similar to those in Step 1, except the fact that we have to use the integral estimates from Theorem 3.3 rather than Corollary 3.4.

First, for any $n=1,2, \ldots$, we let $b_{n}:=b \vee 1 / n$. Then, for any fixed $n$, the function $b_{n}$ is bounded from above and away from zero. By Step 1, for any $n=1,2, \ldots$, there exists a complete probability space $\left(\Omega^{n}, \mathcal{F}^{n}, P^{n}\right)$ with a filtration $\mathbf{F}^{n}$ and the processes $\left(X^{n}, Z^{n}\right)$ on it so that $\left(Z^{n}, \mathbf{F}^{n}\right)$ is a symmetric stable process of index $\alpha$ and it holds

$$
X_{t}^{n}=x_{0}+\int_{0}^{t} b_{n}\left(X_{s-}^{n}\right) d Z_{s}^{n}+\gamma \int_{0}^{t}\left|b_{n}\right|^{\alpha}\left(X_{s}^{n}\right) d s \quad t \geq 0 \quad P^{n} \text {-a.s. }
$$

Similarly as in Step 1, we can verify that the processes $\left(X^{n}, Z^{n}\right), n=1,2, \ldots$ will satisfy two properties (4.4) and (4.5) from the Aldous' criterion where we have to use $P^{n}$ instead of $P$. Therefore, the sequence $\left(X^{n}, Z^{n}\right)$ is tight in the sense of weak convergence in the space $\left(\mathbf{D}^{2}, \mathcal{D}^{2}\right)$ so that one will be able to find a probability space $(\tilde{\Omega}, \tilde{\mathcal{F}}, \tilde{P})$ and processes $\left(\tilde{X}^{n}, \tilde{Z}^{n}\right)$ and $(\tilde{X}, \tilde{Z})$ defined on it so that

i) $\left(\tilde{X}^{n}, \tilde{Z}^{n}\right) \rightarrow(\tilde{X}, \tilde{Z})$ as $n \rightarrow \infty \tilde{P}$-a.s.

ii) $\left(\tilde{X}^{n}, \tilde{Z}^{n}\right)=\left(X^{n}, Z\right)$ in distribution for all $n=1,2, \ldots$

Additionally, using the same arguments as in Step 1, one can establish that

$$
\tilde{X}_{t}^{n}=x_{0}+\int_{0}^{t} b_{n}\left(\tilde{X}_{s}^{n}\right) d \tilde{Z}_{s}^{n}+\gamma \int_{0}^{t}\left|b_{n}\right|^{\alpha}\left(\tilde{X}_{s}^{n}\right) d s, \quad t \geq 0, \quad \tilde{P} \text {-a.s. }
$$

and (4.6) holds.

Therefore, to show that the process $\tilde{X}$ is a solution of the equation (1.5), it suffices to verify that, for all $t \geq 0$, the relations (4.7) and (4.8) hold. In turn, for that it is enough to prove the relation (4.7) only. Moreover, as before, the limiting process $\tilde{X}$ will satisfy the corresponding integral estimate (4.9) from Lemma 4.2 of the form

$$
\tilde{\mathbf{E}} \int_{0}^{t \wedge \tau_{m_{k}}(\tilde{X})} f\left(\tilde{X}_{s}\right) d s \leq M\left\|f|b|^{-\alpha}\right\|_{2, m_{k}}
$$

since

$$
f\left(\tilde{X}_{s}\right)=e^{\phi_{s}} e^{-\phi_{s}}|b|^{\alpha}\left(\tilde{X}_{s}\right)\left[|b|^{-\alpha} f\right]\left(\tilde{X}_{s}\right) \leq M_{4} e^{-\phi_{s}}|b|^{\alpha}\left(\tilde{X}_{s}\right)\left[|b|^{-\alpha} f\right]\left(\tilde{X}_{s}\right)
$$

and the constant $M_{4}$ depends on $K, m$, and $t$ only.

Now, to prove (4.7), it is enough to verify that, for all $t \geq 0$ and $\varepsilon>0$, we have

$$
\lim _{n \rightarrow \infty} \tilde{P}\left(\left|\int_{0}^{t} b_{n}\left(\tilde{X}_{s}^{n}\right) d \tilde{Z}_{s}^{n}-\int_{0}^{t} b\left(\tilde{X}_{s}\right) d \tilde{Z}_{s}\right|>\varepsilon\right)=0 .
$$

In order to prove (4.15), we first estimate for a fixed $k \in \mathbb{Z}_{+}$

$$
\tilde{P}\left(\left|\int_{0}^{t} b_{n}\left(\tilde{X}_{s}^{n}\right) d \tilde{Z}_{s}^{n}-\int_{0}^{t} b\left(\tilde{X}_{s}\right) d \tilde{Z}_{s}\right|>\varepsilon\right) \leq
$$




$$
\begin{gathered}
\tilde{P}\left(\left|\int_{0}^{t} b_{k}\left(\tilde{X}_{s}^{n}\right) d \tilde{Z}_{s}^{n}-\int_{0}^{t} b_{k}\left(\tilde{X}_{s}\right) d \tilde{Z}_{s}\right|>\frac{\varepsilon}{3}\right) \\
+\tilde{P}\left(\left|\int_{0}^{t \wedge \tau_{m}\left(\tilde{X}^{n}\right)}\left[b_{k}-b_{n}\right]\left(\tilde{X}_{s}^{n}\right) d \tilde{Z}_{s}^{n}\right|>\frac{\varepsilon}{3}\right) \\
+\tilde{P}\left(\left|\int_{0}^{t \wedge \tau_{m}(\tilde{X})}\left[b_{k}-b\right]\left(\tilde{X}_{s}\right) d \tilde{Z}_{s}\right|>\frac{\varepsilon}{3}\right)+\tilde{P}\left(\tau_{m}\left(\tilde{X}^{n}\right)<t\right)+\tilde{P}\left(\tau_{m}(\tilde{X})<t\right) \leq \\
\tilde{P}\left(\left|\int_{0}^{t} b_{k}\left(\tilde{X}_{s}^{n}\right) d \tilde{Z}_{s}^{n}-\int_{0}^{t} b_{k}\left(\tilde{X}_{s}\right) d \tilde{Z}_{s}\right|>\frac{\varepsilon}{3}\right) \\
+I_{n, k, m}^{1}+I_{k, m}^{2} \\
+\tilde{P}\left(\tau_{m}\left(\tilde{X}^{n}\right)<t\right)+\tilde{P}\left(\tau_{m}(\tilde{X})<t\right) .
\end{gathered}
$$

To show that $I_{n, k, m}^{1} \rightarrow 0$ as $n \rightarrow \infty$ and $I_{k, m}^{2} \rightarrow 0$ as $k \rightarrow \infty$, we use first the inequality (4.2) and then the Theorem 3.3 and estimate (4.14) for the limiting process, respectively, to obtain

$$
I_{n, k, m}^{1} \leq \varepsilon^{-\alpha} M_{1}\left\|\left|b_{n}-b_{k}\right|^{\alpha} b_{n}^{-\alpha}\right\|_{2, m}
$$

and

$$
I_{k, m}^{2} \leq \varepsilon^{-\alpha} M_{2}\left\|\left|b-b_{k}\right|^{\alpha} b^{-\alpha}\right\|_{2, m}
$$

where the constants $M_{1}$ and $M_{2}$ depend on $\alpha, m$, and $t$ only.

Now, the obvious inequalities

$$
\left(b_{n}-b_{k}\right)^{2 \alpha} b_{n}^{-2 \alpha} \leq\left[2\left(b_{n}^{2}+b_{k}^{2}\right) b_{n}^{-2}\right]^{\alpha} \leq 2^{\alpha}\left(1+b_{k}^{2} b_{n}^{-2}\right)^{\alpha}
$$

and

$$
b_{n}^{-1} \leq b^{-1} \vee 1 / n
$$

combined with the condition $|b|^{-2 \alpha} \in L^{\text {loc }}$ imply that the sequence of functions $\left|b_{n}-b_{k}\right|^{2 \alpha}\left|b_{n}\right|^{-2 \alpha}, n=1,2, \ldots$ is uniformly integrable on $[-m, m]$ so that $\mid b_{n}-$ $\left.b_{k}\right|^{2 \alpha}\left|b_{n}\right|^{-2 \alpha} \rightarrow\left|b-b_{k}\right|^{2 \alpha}|b|^{-2 \alpha}$ as $n \rightarrow \infty$ in $L_{1}[-m, m]$. Thus, the term $I_{n, k, m}^{1}$ converges to zero as $n \rightarrow \infty$ and $k \rightarrow \infty$. The convergence of the term $I_{k, m}^{2}$ to zero as $k \rightarrow \infty$ then follows.

We now consider the term

$$
\tilde{P}\left(\left|\int_{0}^{t} b_{k}\left(\tilde{X}_{s}^{n}\right) d \tilde{Z}_{s}^{n}-\int_{0}^{t} b_{k}\left(\tilde{X}_{s}\right) d \tilde{Z}_{s}\right|>\frac{\varepsilon}{3}\right) .
$$

For any fixed $k$, we can find a sequence of Lipshitz continuous, uniformly bounded functions $b_{k, l}, l=1,2, \ldots$ so that $\left\|b_{k, l}-b_{k}\right\|_{2, m} \rightarrow 0$ for any $m$ as $l \rightarrow \infty$. Using this fact, we can estimate

$$
\begin{gathered}
\tilde{P}\left(\left|\int_{0}^{t} b_{k}\left(\tilde{X}_{s}^{n}\right) d \tilde{Z}_{s}^{n}-\int_{0}^{t} b_{k}\left(\tilde{X}_{s}\right) d \tilde{Z}_{s}\right|>\frac{\varepsilon}{3}\right) \leq \\
\tilde{P}\left(\left|\int_{0}^{t} b_{k, l}\left(\tilde{X}_{s}^{n}\right) d \tilde{Z}_{s}^{n}-\int_{0}^{t} b_{k, l}\left(\tilde{X}_{s}\right) d \tilde{Z}_{s}\right|>\frac{\varepsilon}{9}\right) \\
+\tilde{P}\left(\left|\int_{0}^{t \wedge \tau_{m}\left(\tilde{X}^{n}\right)}\left[b_{k, l}-b_{k}\right]\left(\tilde{X}_{s}^{n}\right) d \tilde{Z}_{s}^{n}\right|>\frac{\varepsilon}{9}\right) \\
+\tilde{P}\left(\left|\int_{0}^{t \wedge \tau_{m}(\tilde{X})}\left[b_{k, l}-b_{k}\right]\left(\tilde{X}_{s}\right) d \tilde{Z}_{s}\right|>\frac{\varepsilon}{9}\right)+\tilde{P}\left(\tau_{m}\left(\tilde{X}^{n}\right)<t\right)+\tilde{P}\left(\tau_{m}(\tilde{X})<t\right)=
\end{gathered}
$$




$$
J_{k, l, n}^{1}+J_{k, l, n, m}^{2}+J_{k, l, m}^{3}+\tilde{P}\left(\tau_{m}\left(\tilde{X}^{n}\right)<t\right)+\tilde{P}\left(\tau_{m}(\tilde{X})<t\right) .
$$

Proposition 2.2 implies now that the term $J_{k, l, n}^{1}$ converges to zero as $n \rightarrow \infty$. The terms $J_{k, l, n, m}^{2}$ and $J_{k, l, m}^{3}$ can be estimated by the corresponding integral estimates as

$$
J_{k, l, n, m}^{2} \leq \varepsilon^{-\alpha} M_{1}\left\|\left|b_{k, l}-b_{k}\right|^{\alpha} b_{n}^{-\alpha}\right\|_{2, m}
$$

and

$$
J_{k, l, m}^{3} \leq \varepsilon^{-\alpha} M_{2}\left\|\left|b_{k, l}-b_{k}\right|^{\alpha} b^{-\alpha}\right\|_{2, m}
$$

where the constants $M_{1}$ and $M_{2}$ depend on $\alpha, m$, and $t$ only. By letting $l \rightarrow \infty$, both terms $J_{k, l, n, m}^{2}$ and $J_{k, l, m}^{3}$ will converge to zero.

Finally, because of the property $\tau_{m}\left(\tilde{X}^{n}\right) \rightarrow \tau_{m}(\tilde{X})$ as $n \rightarrow \infty \tilde{P}$-a.s.,

$$
\tilde{P}\left(\tau_{m}\left(\tilde{X}^{n}\right)<t\right) \rightarrow \tilde{P}\left(\tau_{m}(\tilde{X})<t\right) \quad \text { as } n \rightarrow \infty
$$

for all $m \in \mathbb{Z}_{+}, t>0$. Thus, the convergence to zero of the last two terms $\tilde{P}\left(\tau_{m}\left(\tilde{X}^{n}\right)<t\right)$ and $\tilde{P}\left(\tau_{m}(\tilde{X})<t\right)$ can be justified in a similar form as in Step 1.

We use now Theorem 4.1 to prove the existence of solutions for the equation (1.5) with $\gamma=0$.

Theorem 4.3. Let $0<\alpha<2$. Assume that $|b|^{-2 \alpha} \in L^{l o c}(\mathbb{R}),|b(x)| \leq K$ for all $x \in \mathbb{R}$ where $K>0$ is a constant, and $b(x)$ is a continuous function in $x$. Then, for any $x_{0} \in \mathbb{R}$, there exists a solution of the equation (1.5) without drift.

Proof: We choose a sequence of real numbers $\gamma_{n} \neq 0, n=1,2, \ldots$. such that $\lim _{n \rightarrow \infty} \gamma_{n}=0$ and consider the stochastic equation

$$
d X_{t}^{n}=b\left(X_{t-}^{n}\right) d Z_{t}^{n}+\gamma_{n}|b|^{\alpha}\left(X_{t}^{n}\right) d t, \quad t \geq 0, \quad X_{0}^{n}=x_{0} \in \mathbb{R}
$$

where $Z^{n}$ is a symmetric stable process of index $0<\alpha<2$.

For any fixed $n=1,2, \ldots$, the conditions of Theorem 4.1 are satisfied. Therefore, the equation (4.18) has a (weak) solution. In other words, there exists a complete probability space $\left(\Omega^{n}, \mathcal{F}^{n}, P^{n}\right)$ and the processes $\left(X^{n}, Z^{n}\right)$ on it so that $Z^{n}$ is a symmetric stable process of index $\alpha$ and (4.18) holds.

Now, consider the sequence of processes $\left(J^{n}, X^{n}, Z^{n}\right), n \geq 1$, where

$$
J_{t}^{n}=\gamma_{n} \int_{0}^{t}|b|^{\alpha}\left(X_{s}^{n}\right) d s, t \geq 0 .
$$

Since $b$ is bounded, the sequence of processes $\left(J^{n}, X^{n}, Z^{n}\right), n \geq 1$ will satisfy the Aldous' conditions (4.4) and (4.5) hence is tight in the sense of weak convergence in $\left(\mathbf{D}^{3}, \mathcal{D}^{3}\right)$. Also, by the same argument, the pair $\left(J^{n}, Z^{n}\right)$ will converge in distribution to $(J, Z)$ where $J=0$ and $Z$ is a symmetric stable process of index $\alpha$.

We also notice that the functional $b(z(t))$ is Skorokhod continuous on the space $\mathbf{D}$ because the function $b$ is assumed to be continuous and, for each $t>0$, the sequence $\left\{\int_{0}^{t} H_{s-}^{n} d Z_{s}^{n}\right\}$ is stochastically bounded (uniformly in $n$ ) where $H^{n}$ is any predictable simple process such that $\left|H^{n}\right| \leq 1, n \geq 1$. The latter follows from the inequality (4.2).

According to the Theorem of Th. Kurtz and Ph. Protter about the convergence of solutions of stochastic differential equations with respect to semimartingales (see Proposition 5.1 in Kurtz and Protter, 1991), any limiting point of the 
sequence $\left(X^{n}\right)$ will then satify the equation

$$
d X_{t}=b\left(X_{t-}\right) d Z_{t}, x_{0} \in \mathbb{R}, t \geq 0 .
$$

Thus, the existence of a solution of equation (1.5) without drift is proven.

Example 4.4. Let

$$
b(x)= \begin{cases}-1, & x<-1 \\ x, & x \in[-1,1] \\ 1, & x>1\end{cases}
$$

and assume $0<\alpha<1 / 2$. The function $b(x)$ satisfies the assumptions of the Theorem 4.3 so that there is a solution of the equation (1.5). At the same time, the coefficient $b$ does not satisfy the conditions found by Zanzotto (see Introduction). Moverover, Theorem 4.3 also improves the local integrability condition on the coefficient $b$ found by Zanzotto for the SDE without drift in case of $0<\alpha<1 / 2$ in general. Contrary to the condition $|b|^{-\delta} \in L^{l o c}$ with $\delta>1$ required in the existence theorem of Zanzotto, Theorem 4.3 assumes only $|b|^{-\delta} \in L^{l o c}$ which is satisfied with $\delta=2 \alpha<1$ since $0<\alpha<1 / 2$.

\section{Acknowledgements}

The author would like to thank the anonymous referee for the careful reading of the manuscript and helpful remarks which improved the paper.

\section{References}

D. Aldous. Stopping times and tightness. Ann. Probability 6 (2), 335-340 (1978). MR0474446.

S. Anulova and G. Pragarauskas. Weak Markov solutions of stochastic equations. Litovsk. Mat. Sb. 17 (2), 5-26, 219 (1977). MR0651573.

D. Appelbaum. Levy processes and stochastic calculus. Cambridge University Press (2009). Second edition. MR2512800.

C. Dellacherie and P.A. Meyer. Probabilities et Potentiels. Publications de l'Institut de Mathmatique de l'Université de Strasbourg (1980). First edition. MR0488194.

H. J. Engelbert and W. Schmidt. Strong Markov continuous local martingales and solutions of one-dimensional stochastic differential equations. III. Math. Nachr. 151, 149-197 (1991). MR1121203.

N. Ikeda and S. Watanabe. Stochastic differential equations and diffusion processes, volume 24 of North-Holland Mathematical Library. North-Holland Publishing Co., Amsterdam-New York; Kodansha, Ltd., Tokyo (1981). ISBN 0-444-86172-6. MR637061.

O. Kallenberg. Foundations of modern probability. Probability and its Applications (New York). Springer-Verlag, New York (1997). ISBN 0-387-94957-7. MR1464694.

N. V. Krylov. Controlled diffusion processes, volume 14 of Applications of Mathematics. Springer-Verlag, New York-Berlin (1980). ISBN 0-387-90461-1. MR601776.

V. Kurenok. A note on $L_{2}$-estimates for stable integrals with drift. Trans. Amer. Math. Soc. 360 (2), 925-938 (2008a). MR2346477. 
V. Kurenok. Stochastic equations driven by a Cauchy process. In Markov processes and related topics: a Festschrift for Thomas G. Kurtz, volume 4 of Inst. Math. Stat. (IMS) Collect., pages 99-106. Inst. Math. Statist., Beachwood, OH (2008b). MR2574226.

T. G. Kurtz and P. Protter. Weak limit theorems for stochastic integrals and stochastic differential equations. Ann. Probab. 19 (3), 1035-1070 (1991). MR1112406.

J.-P. Lepeltier and B. Marchal. Problème des martingales et équations différentielles stochastiques associées à un opérateur intégro-différentiel. Ann. Inst. H. Poincaré Sect. B (N.S.) 12 (1), 43-103 (1976). MR0413288.

A. V. Mel'nikov. Stochastic equations and Krylov's estimates for semimartingales. Stochastics 10 (2), 81-102 (1983). MR716817.

G. Pragarauskas and P. A. Zanzotto. On one-dimensional stochastic differential equations with respect to stable processes. Liet. Mat. Rink. 40 (3), 361-385 (2000). MR1803652.

J. Rosiński and W. A. Woyczyński. On Itô stochastic integration with respect to p-stable motion: inner clock, integrability of sample paths, double and multiple integrals. Ann. Probab. 14 (1), 271-286 (1986). MR815970.

R. Situ. Theory of stochastic differential equations with jumps and applications. Mathematical and Analytical Techniques with Applications to Engineering. Springer, New York (2005). ISBN 978-0387-25083-0; 0-387-25083-2. MR2160585.

P. A. Zanzotto. On solutions of one-dimensional stochastic differential equations driven by stable Lévy motion. Stochastic Process. Appl. 68 (2), 209-228 (1997). MR1454833.

P. A. Zanzotto. On stochastic differential equations driven by a Cauchy process and other stable Lévy motions. Ann. Probab. 30 (2), 802-825 (2002). MR1905857. 